\section{Stratification might be appropriate for patients with node-positive uterine cancer}

An association between the extent of lymph node involvement and prognosis is well documented in many solid tumors, including lung, breast and cervical cancer. Yet, the International Federation of Gynecology and Obstetrics staging system for uterine cancer does not take into account the extent of nodal disease. Now, Chan and co-workers present data indicating that stratification of patients with node-positive uterine cancer might be appropriate.

The researchers identified 1,222 women with stage IIIC-IV node-positive endometrioid corpus cancer, from data obtained by the National Cancer Institute registry from 1998 to 2001. Patients were stratified according to ratio of positive lymph nodes-expressed as a percentage of positive nodes to total nodes identified-and absolute number of positive lymph nodes. Increasing positive-lymph-node ratio and absolute number of positive lymph nodes were associated with decreased survival, regardless of disease stage and extent of lymphadenectomy. Patients with positive-lymph-node ratios of $\leq 10 \%,>10-50 \%$ and $>50 \%$ had 5 -year disease-specific survival rates of $77.3 \%$, $60.7 \%$ and $40.9 \%$, respectively $(P<0.001$ for trend), while patients with $1,2-5$ and $>5$ positive nodes had 5-year disease-specific survival rates of $68.1 \%, 55.1 \%$ and $46.1 \%$, respectively $(P<0.001$ for trend). Multivariate analysis revealed that both absolute number of positive nodes and positive-lymph-node ratio were independent predictors of survival. The authors conclude that further investigations into the stratification of patients with node-positive uterine cancer are warranted.

Original article Chan JK et al. (2007) The impact of the absolute number and ratio of positive lymph nodes on survival of endometrioid uterine cancer patients. Br J Cancer 97: 605-611

\section{Comorbid conditions do not negate the benefit of adjuvant chemotherapy in colon cancer}

Adjuvant chemotherapy for colon cancer reduces mortality in patients of all ages; however, several studies have reported an inverse correlation between age and receipt of adjuvant therapy. The increased burden of chronic disease in elderly patients with cancer might explain the lower use of adjuvant therapy in this population. To determine the effect of comorbid conditions on the use and effectiveness of adjuvant chemotherapy in colon cancer, Gross et al. conducted a cohort study comprising 5,330 patients (median age 76 years) with stage III disease.

Overall, approximately $44.7 \%$ of the study population had 1 or 2 comorbid conditions and $8.8 \%$ had 3 or more. The probability of receiving chemotherapy was inversely correlated with age and number of comorbid conditions. Patients with heart failure were significantly less likely to receive chemotherapy than were patients without this condition (adjusted odds ratio $[\mathrm{OR}] 0.49,95 \% \mathrm{Cl} 0.40-0.60 ; P<0.001$ ). The probability of receiving adjuvant therapy was also reduced in patients with chronic pulmonary disease (OR $0.83,95 \% \mathrm{Cl} 0.70$ 0.99; $P=0.04$ ) or diabetes (OR $0.8195 \% \mathrm{Cl}$ $0.68-0.97 ; P=0.02)$. Patients who received adjuvant therapy had higher rates of 5-year overall survival than did untreated patients, and this relationship held true when patients were stratified according to comorbid condition. The risks of all-cause, condition-specific or toxicity-related hospitalization were not influenced by the presence of heart failure, chronic obstructive pulmonary disease or diabetes. The authors suggest that further study to explore the effectiveness of adjuvant therapy in the presence of comorbidity is greatly needed.

Original article Gross CP et al. (2007) The impact of

chronic illnesses on the use and effectiveness of adjuvant chemotherapy for colon cancer. Cancer 109: 2410-2419

\section{Better survival in patients with ovarian cancer referred to gynecologic oncologists}

Guidelines recommend that women with pelvic masses suspicious for ovarian cancer are referred to physicians with adequate skill in both surgery and treatment. Nevertheless, as reported by Chan et al., many women are not referred to gynecologic oncologists and consequently may not receive the best treatment, with a deleterious effect on their outcome (level II evidence).

The group reviewed data from 1,491 women with primary invasive epithelial ovarian cancer from the population-based California 